\title{
The effects of nanoemulsions on the fatty acid profiles of sea bass fillets during storage at $2 \pm 2{ }^{\circ} \mathrm{C}$
}

\section{Nanoemüllsiyonların $2 \pm 2^{\circ} \mathrm{C}^{\prime} \mathrm{de}$ depolanan levrek filetolarının yağ asidi profilleri üzerine etkileri}

\author{
Mustafa Durmuş ${ }^{1^{*}}$ (D) Yeşim Özoğul ${ }^{1}$ \\ 1 Department of Seafood Processing Technology, Faculty of Fisheries, Çukurova University, Adana, Turkey
}

*Corresponding author: mdurmus@cu.edu.tr

How to cite this paper:

Durmuş, M. \& Özoğul, Y. (2018). The effects of nanoemulsions on the fatty acid profiles of sea bass fillets during storage at $2 \pm 2{ }^{\circ} \mathrm{C}$. Ege Journal of Fisheries and Aquatic Sciences, 35(3), 227-235. DOI:10.12714/egejfas.2018.35.3.01

\begin{abstract}
The effect of nanoemulsions prepared from commercial oils on the fatty acid profiles of cold-stored sea bass fillets was investigated. The sea bass fillets were treated with nanoemulsions prepared from sunflower, canola, corn, olive, soybean, and hazelnut oils (14\% of the total emulsion). Results of fatty acid analyses showed that the main fatty acids found in all groups were determined to be miristic acid, palmitic acid, stearic acid, palmitoleic acid, oleic acid, linoleic acid, eicosapentaenoic acid (EPA) and docosahexaenoic acid (DHA). At the end of storage, the control group (24.05\%) had the highest SFA content, while the lowest SFA among the nanoemulsion treatment groups was observed in the olive group (21.97\%) followed by sunflower group (22.78\%). The oleic (C18:1n9) and palmitoleic acids (C16:1) from monounsaturated fatty acids (MUFA) varied between $30.45-23.82 \%$ and $4.92-3.42 \%$ during storage period. Among all polyunsaturated fatty acids (PUFA), linoleic acid, EPA and DHA were predominant fatty acids. EPA and DHA ranged from 4.73 to $2.81 \%$ and 8.09 to $4.06 \%$, respectively. While the lowest PUFA content was observed in the control group, the highest value was determined in the soybean group (26.41\%), followed by olive $(24.48 \%)$ and canola $(24.46 \%)$ at the end of storage. The results showed that the nanoemulsion application delayed lipid oxidation. Application of nanoemulsion compared to control group maintained the PUFA content of fish and can be used as a preservative for fish.
\end{abstract}

Keywords: Nanoemulsion, sea bass, fatty acids, EPA, DHA

Öz: Ticari yağlardan hazırlanan nanoemülsiyonların soğukta depolanmış levrek filetolarının yağ asit profilleri üzerindeki etkileri araştırımışıı. Levrek filetoları, ayçiçeği, kanola, mısır, zeytin, soya ve fındık yağlarından hazırlanan nanoemülsiyonlar ile muamele edildi (toplam emülsiyonun \% 14 'ü). Yağ asidi analiz sonuçlarına göre, tüm gruplarda bulunan temel yağ asitlerinin, miristik asit, palmitik asit, stearik asit, palmitoleik asit, oleik asit, linoleik asit, eikosapentaenoik asit (EPA) ve dokosaheksaenoik asit (DHA) olduğu belirlendi. Depolama sonunda, kontrol grubu en yüksek SFA (\%24.05) içeriğine sahip iken, nanoemülsiyon uygulanan gruplar arasında ise en düşük SFA zeytin grubunda (\%21.97), ardından ayçiçeği grubunda (\%22.78) gözlenmiştir. Tekli doymamış yağ asitleri (MUFA) 'den oleik asit (C18: 1n9) ve palmitoleik asit (C16: 1) miktarları depolama süresi boyunca sırasıyla \%30.45-23.82 ve \%4.92-3.42 aralığında değişmiştir. Çoklu doymamıs yağ asitleri (PUFA) arasında linoleik asit, EPA ve DHA yağ asitteri en yüksek değere sahip olduğu belirlendi. EPA ve DHA miktarları sırasıyla \% $4.73-$ 2.81 ve \% 8.09-4.06 aralıklarında oldukları belirlenmiştir. Depolama sonunda en düşük PUFA içeriği kontrol grubunda gözlenirken, en yüksek değer soya grubunda (\%26.41), bunu takiben zeytin (\%24.48) ve kanola (\%24.46) gruplarında gözlenmiştir. Sonuçlar nanoemülsiyon uygulamasının lipid oksidasyonunu geciktirdiğini göstermiştir. Kontrol grubu ile kıyasladığımızda nanoemülsiyon uygulamasının, balıkların PUFA içeriğini muhafaza ettiğini ve bir koruyucu olarak kullanılabileceğini göstermiştir.

Anahtar kelimeler: Nanoemülsiyon, levrek, yağ asitleri, EPA, DHA

\section{INTRODUCTION}

Fish oil contains essential fatty acids like eicosapentaenoic acid (EPA) and docosahexaenoic acid (DHA), which makes consumption of fish more important for human health. It is known that these essential fatty acids have an important role in preventing many diseases such as heart attacks, cardiovascular diseases, depression, migraine, rheumatism of the joints, diabetes, high cholesterol, hypertension, cancers and allergies (Kinsella, 1987; Leaf et al., 1988; Simopoulos,
1991; Rendeiro et al., 2016; Palmquist, 2009; Stoll, 2002; Itsiopoulos et al., 2009). In addition, omega fatty acids have an important role in the development of the brain and the immune system. Low DHA level in brain cells can lead to problems like depression, memory loss, Alzheimer's, schizophrenia and vision problems (Kaya et al., 2004). Children who consume fish at least twice a week have a lower chance of developing emotional and behavioural disorders (Llaurado et al., 2016). 
Fatty acids (especially unsaturated fatty acids) are the main resource for the production of volatile compounds ( $\mathrm{Ba}$ et al. 2013). Fatty acid composition affects fish meat's sensory quality and lipid stability (Nuernberg et al., 2005). Fish oils are affected from the marine environment and the seasonal changes in the water temperature and food availability. These are the most important factors influencing the nutritional composition of fish. The taste of fish meat is closely associated with the protein and fat content, and the knowledge on the fatty acid profile of fish especially important to predict the positive effects of fish meat consumption on human health.

Lipid oxidation is not desired in most foodstuffs, as it can lead to spoilage and unwanted tastes that are caused by toxic reaction products (Halliwell et al. 1995). Lipid oxidation involves a reaction between unsaturated lipids and oxygen. Oxygen dissolves in food fats 3 times as much as it does in water (Ke \& Ackman, 1973). Synthetic or natural antioxidants can be used to delay lipid oxidation. Lately use of natural antioxidants is preferred by both the consumers and the food industry. As a consequence, new methods that allow the use of safer and more effective natural materials that can replace synthetic methods has increased. Recently, nanotechnology has become one of the methods that are used for food preservation.

The use of nanotechnology in food industry can be classified under three categories: nutritional enrichment of foods, protection of food and development of new products. An important variety of nanotechnology applications in food is nanoemulsions. Nanoemulsions are transparent or semitransparent emulsions that contain nano-scale droplets. Nanoemulsions are nano droplets that consist of multiphase colloidal (homogenous looking heterogenous mixture) distributions that are created by taking two liquid that don't physically mix together and distributing one within the other (Fathi et al., 2012; Liu et al., 2006; Mason et al., 2006; Meleson et al., 2004; Russel et al., 1989). Different size ranges were given for nanoemulsions in literature; for example size ranges of below $100 \mathrm{~nm}$ (Guo et al., 2007; Porras et al., 2008; Shakeel et al., 2010), between 10-100 nm (Talegaonkar et al., 2010), between 100-500 nm (Anton et al., 2008; Constantinides et al., 2008; Rossi et al., 2007; Tadros et al., 2004; Ungeret et al., 2004) and between 100-600nm (Sakulku et al., 2009; Solans et al., 2003).

Nanoemulsions are also known as antimicrobial preservatives. The reason is that they connect the structure of water and thereby limit the access to water of microorganisms (Al-Adham et al., 2000). Many studies demonstrated that nanoemulsions have a preventative effect on bacteria (Friberg, 1984; Jones et al. 1997; Bortoleto et al.,1998), fungi (Hamouda et al., 1999; Myc et al., 2001; Zhang et al., 2008) and viruses (Donovan et al., 2000; Chepurnov et al., 2003). Nanoemulsions are not just kinetically stable, they are also physically stable in the long term (Bouchernal et al, 2004). Nanoemulsions can also be considered self-protecting antimicrobials (Al-Adham et al, 2000). Furthermore, it was reported that nanoemulsions delay lipid oxidation (Ozoğul et al
2016; Durmus, 2016). Yazgan (2013) reported that sunflower oil nanoemulsion reduce the lipid oxidation parameters in sea bass and sea bream fillets. In our previous study (Ozogul et al., 2017), the combined impact of nanoemulsion based on commercial oils and vacuum packing on the fatty acid profiles of sea bass fillets was investigated. Vacuum packaging and nanoemulsion were reported to have a positive effect on fatty acids in sea bass fillets (Ozogul et al. 2017). Shadman et al. (2016) reported that sunflower oil nanoemulsions could prevent lipid oxidation in rainbow trout fillets during storage. The aim of present study was to investigate the effects of nanoemulsion groups prepared using commercially oils (sunflower oil, hazelnut oil, canola oil, soybean oil, corn oil and olive oil) on the fatty acid profiles of cold stored sea bass fillets.

\section{MATERIALS AND METHODS}

\section{Preparation of nanoemulsions}

Nanoemulsions were prepared according to the method of Joe et al. (2012) with minor modification. Oils used were purchased from the local market (Adana, Turkey). The oil phase of the oil-in-water $(\mathrm{O} / \mathrm{W})$ nanoemulsions consists of commercial oil (sunflower (S), canola (Can), corn, olive (O), soybean (SB), and hazelnut oils $(\mathrm{H}) ; 14 \%$ of the total emulsion), ethanol $(3 \%)$, and a surfactant (Tween $80,3 \%$, generally regarded as safe (GRAS)) (Sigma-Aldrich, Lyon, France) and represents $20 \%(\mathrm{v} / \mathrm{v})$ of the emulsion. The components of this oil phase were mixed and kept for $1 \mathrm{~h}$ at $86^{\circ} \mathrm{C}$ and after which the compounds were mixed with water $(80 \%)$. The mixture was homogenised using an ultrasonic homogeniser (Optic Ivymen System CY-500, Barcelona, Spain) for $15 \mathrm{~min}$. Properties of the oil-in-water nanoemulsions based on commercial oils were given in our previous study (Ozogul et al., 2016).

\section{Sample preparation}

Sea bass (D. labrax) were obtained from a local fish farm in İmir, Turkey. Fish were killed by dipping in ice-cold water (hypothermia). After death, the fish were transported to the laboratory in ice within 24 to $25 \mathrm{~h}$ from harvesting. The average length and weight of the sea bass were $29.7 \pm 1.0 \mathrm{~cm}$ and 270 $\pm 23 \mathrm{~g}$. They were immediately gutted, filleted with skin on, and divided into seven lots. The control group $(C)$ was stored on plates (6 fillets per plate) wrapped with permeable stretch film (Prima Gida Iht. Mad. Ambalaj. San, Mersin, Turkey). The other samples were treated with nanoemulsions. Fish fillets were immersed for $4 \mathrm{~min}$ in the nanoemulsions prepared with different oils and then stored 6 fillets to a plate wrapped with stretch film. The average sea bass fillet weight was $55 \pm 7 \mathrm{~g}$. For each analysis day, 3 plates (total 18 fillets) were randomly selected for each group. All samples were stored in a chill room $\left(2 \pm 2{ }^{\circ} \mathrm{C}\right)$. Data were obtained from fillets from three plates (triplicate) treated separately with duplicate measurements of the appropriate sample from each plate $(n=6)$.

\section{Fatty acid analyses}

Lipid content was determined by the method of Bligh \& Dyer (1959). Lipid samples were converted to their constituent fatty 
acid methyl esters by the method of Ichihara et al. (1996), by using $2 \mathrm{M} \mathrm{KOH}$ in methanol and n-heptane with minor modifications. Twenty $\mathrm{mg}$ of extracted oil was dissolved in $2 \mathrm{ml}$ n-heptane followed by $4 \mathrm{ml}$ of $2 \mathrm{M}$ methanolic $\mathrm{KOH}$. The tube was then vortexed for $2 \mathrm{~min}$ at room temperature. After centrifugation at $4,000 \mathrm{rpm}$ for $10 \mathrm{~min}$, the $\mathrm{n}$-heptane layer was taken for gas chromatography analyses.

\section{Statistical analysis}

SPSS 22.0 was used to determine for statistical analysis. Data obtained from quality parameters of sea bass fillets treated with nanoemulsions during storage carried out with Duncan.

\section{RESULTS AND DISCUSSION}

The main fatty acids observed in all groups were miristic acid $\left(\mathrm{C}_{14: 0}\right)$, palmitic acid $\left(\mathrm{C}_{16: 0}\right)$, stearic acid $\left(\mathrm{C}_{18: 0}\right)$, palmitoleic acid $\left(C_{16: 1}\right)$, oleic acid $\left(C_{18: 1 n 9}\right)$, linoleic acid $\left(C_{18: 2 n 6}\right)$, eicosapentaenoic acid (EPA, $\mathrm{C}_{20: 5} \mathrm{n} 3$ ) and docosahexaenoic

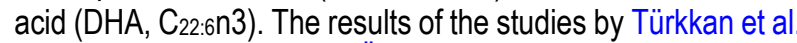
(2008), Yıldız et al. (2008), Özden et al. (2010), Yazgan (2013) and Ozogul et al. (2017) on sea bass were in agreement with the results of the current study.

The changes in saturated fatty acid (SFA) in sea bass fillets treated with nanoemulsions and stored in cold storage were given in Table 1. SFA was found to be $19.21 \%$ at the beginning of storage, and $24.05 \%$ in the control group (untreated group) at the end of the storage period. In groups treated with nanoemulsions, SFA values changed between $19.08 \%$ and $23.43 \%$ during storage. SFA levels increased in all treated groups during storage. At the end of storage, the highest SFA level was found in the canola group with $23.43 \%$ whereas with the lowest SFA found in the olive oil group with $21.97 \%$. Ozogul et al. (2007) reported that the amount of saturated fatty acids in the muscle tissues of commercially valuable freshwater and saltwater fish were in the range of $25.5-39.4 \%$. Baki et al. (2015) reported that the total saturated fat amounts ( $\Sigma$ SFA) in wild and cultured bass (Dicentrarchus labrax) as $26.50 \% \pm 0.06$ and $25.10 \% \pm 0.01$, respectively. Total saturated fatty acid values found in present study were lower than the values given in these studies. Yazgan (2013) determined that the total SFA values for sea bass fillets stored after applying the nanoemulsion prepared with sunflower oil were $21.14 \%$ before storage and $21.73 \%$ after storage.

The main fatty acids among SFA in all groups were miristic acid $\left(\mathrm{C}_{14: 0}\right)$, palmitic acid $\left(\mathrm{C}_{16: 0}\right)$ and stearic acid $\left(\mathrm{C}_{18: 0}\right)$. Miristic acid was determined as $2.65 \%, 2.55 \%, 2.56 \%, 2.61 \%, 2.82 \%$, $2.39 \%, 2.19 \%$ on day 2 of storage and as $3.68 \%, 3.08 \%$, $3.51 \%, 3.37 \%, 3.41 \%, 3.11 \%, 3.08 \%$ on the last day of storage (day 12) for the control, sunflower, hazelnut, canola, soybean, corn and olive oil groups respectively. Fluctuations were observed in miristic acid levels during the storage period. However, increases were also observed in miristic acid level on days 8,10 and 12 of storage $(p<0.05)$. Statistically significant differences were observed between groups during the storage period $(p<0.05)$

Fatty acid with the highest value among SFA was found to be palmitic acid $\left(\mathrm{C}_{16: 0}\right)$. Alasalvar et al. (2002) reported that palmitic acid was an important fatty acid among saturated fatty acids of wild and cultured sea bass. Similar results were reported by Sağlık et al. (2003), Periago et al. (2005), Yazgan (2013) and Ozogul et al. (2017). Palmitic acid level was found as $12.86 \%$ at the beginning of the storage period, while it was determined as $16.50 \%, 15.92 \%, 15.69 \%, 15.79 \%, 15.16 \%$, $15.94 \%$ and $15.08 \%$ at the end of the storage period for the control, sunflower, hazelnut, canola, soybean, corn and olive oil groups respectively. A statistically significant difference was observed between the control group and all nanoemulsion treatment groups on day 2, 4, 6 and 10 of the storage period $(p<0.05)$.

The changes in (MUFA) of sea bass fillets treated with nanoemulsions and stored in cold storage were given in Table 2. A decrease was observed in MUFA levels during the storage period. MUFA level was found as $35.88 \%$ at the beginning of the storage period. While on the second day of storage, sunflower oil nanoemulsion group was found to have the highest MUFA value with $36.30 \%$, the control group gave the lowest MUFA value with $28.80 \%$ on day 12 of the storage period. Ozogul et al. (2017) demonstrated that $\Sigma$ MUFA values for sea bass changed from $41.7 \%$ to $36.41 \%$ during storage period. Baki et al. (2015) reported the total monounsaturated fatty acid values for wild and cultured sea bass as $27.55 \%$ and $30.14 \%$, respectively. Özoğul et al. (2007) found MUFA levels in commercially valuable fish species between 13.2 and $29.0 \%$.

Palmitoleic acid $\left(\mathrm{C}_{16: 1}\right)$ and oleic acid $\left(\mathrm{C}_{18: 1 \mathrm{n} 9}\right)$ among MUFA were main fatty acids in all groups as reported for sea bass by Ozden et al. (2010) and Lenas et al. (2011). The initial palmitoleic acid level was found as $4.71 \%$ and it was changed between $3.42-4.92 \%$ for all groups during the storage period. Over the storage period, statistically significant differences were observed in all groups on every day except day 2 $(p<0.05)$. Oleic acid level was determined as $29.95 \%$ at the beginning of the storage period. Over the storage period, decrease in oleic acid values and statistically significant differences were observed in all groups $(p<0.05)$. At the end of the storage period, the control group gave the lowest oleic acid $(23.82 \%)$, while olive oil group had the highest oleic acid value ( $30.45 \%)$ on day 2 of storage. 
Table 1. Changes in SFA (\%) content of sea bass treated with/without nanoemulsions during storage period

\begin{tabular}{|c|c|c|c|c|c|c|c|c|}
\hline Days & C12:0 & C14:0 & C16:0 & C17:0 & C18:0 & C20:0 & SFA & Groups \\
\hline 0 & $0.44 \pm 0.13$ & $2.50 \pm 0.07$ & $12.86 \pm 0.51$ & $0.09 \pm 0.01$ & $3.18 \pm 0.18$ & $0.15 \pm 0.00$ & $19.21 \pm 0.76$ & C \\
\hline \multirow{7}{*}{2} & $0.93 \pm 0.11^{a}$ & $2.65 \pm 0.08^{\mathrm{ab}}$ & $13.63 \pm 0.01^{a}$ & $0.09 \pm 0.01^{a}$ & $3.13 \pm 0.04^{b}$ & $0.18 \pm 0.01^{\mathrm{a}}$ & $20.60 \pm 0.11 a$ & C \\
\hline & $0.73 \pm 0.01 \mathrm{bc}$ & $2.55 \pm 0.19 \mathrm{ab}$ & $12.72 \pm 0.01^{\mathrm{cd}}$ & $0.08 \pm 0.01 \mathrm{a}$ & $3.24 \pm 0.10^{b}$ & $0.20 \pm 0.05 \mathrm{a}$ & $19.51 \pm 0.16^{b}$ & S \\
\hline & $0.57 \pm 0.00^{c}$ & $2.56 \pm 0.10^{\mathrm{ab}}$ & $13.09 \pm 0.13^{b}$ & $0.08 \pm 0.01^{a}$ & $2.63 \pm 0.06^{c}$ & $0.17 \pm 0.02^{\mathrm{a}}$ & $19.08 \pm 0.33^{b}$ & H \\
\hline & $0.60 \pm 0.07 \mathrm{bc}$ & $2.61 \pm 0.13^{\mathrm{ab}}$ & $12.56 \pm 0.19 \mathrm{de}$ & $0.07 \pm 0.01^{a}$ & $3.32 \pm 0.05^{\mathrm{ab}}$ & $0.16 \pm 0.01^{a}$ & $19.31 \pm 0.46^{b}$ & Can \\
\hline & $0.59 \pm 0.07 \mathrm{bc}$ & $2.82 \pm 0.42^{\mathrm{a}}$ & $12.45 \pm 0.01 \mathrm{e}$ & $0.09 \pm 0.02^{a}$ & $3.33 \pm 0.14 \mathrm{ab}$ & $0.20 \pm 0.01^{\mathrm{a}}$ & $19.47 \pm 0.65^{b}$ & SB \\
\hline & $0.61 \pm 0.04 \mathrm{bc}$ & $2.39 \pm 0.07 \mathrm{ab}$ & $12.81 \pm 0.04^{c}$ & $0.07 \pm 0.01^{a}$ & $3.19 \pm 0.02^{b}$ & $0.17 \pm 0.01^{\mathrm{a}}$ & $19.24 \pm 0.04^{b}$ & Corn \\
\hline & $0.75 \pm 0.05^{b}$ & $2.19 \pm 0.01^{\mathrm{b}}$ & $13.26 \pm 0.01^{b}$ & $0.07 \pm 0.02^{\mathrm{a}}$ & $3.44 \pm 0.07 \mathrm{a}$ & $0.17 \pm 0.01^{a}$ & $19.87 \pm 0.06^{\mathrm{ab}}$ & 0 \\
\hline \multirow{7}{*}{4} & $0.51 \pm 0.04 c$ & $3.36 \pm 0.21^{a}$ & $15.62 \pm 0.33^{a}$ & $0.09 \pm 0.02^{a}$ & $2.69 \pm 0.09 c$ & $0.18 \pm 0.04^{a}$ & $22.43 \pm 0.35^{a}$ & $C$ \\
\hline & $0.50 \pm 0.01^{c}$ & $2.59 \pm 0.13^{b}$ & $12.92 \pm 0.08^{\mathrm{bc}}$ & $0.06 \pm 0.00^{c}$ & $3.36 \pm 0.10^{a}$ & $0.20 \pm 0.06^{a}$ & $19.62 \pm 0.07 \mathrm{bc}$ & $S$ \\
\hline & $0.34 \pm 0.00^{d}$ & $2.56 \pm 0.07^{b}$ & $13.28 \pm 0.14^{\mathrm{bc}}$ & $0.07 \pm 0.00^{\mathrm{ab}}$ & $2.93 \pm 0.13^{b}$ & $0.20 \pm 0.01^{a}$ & $19.37 \pm 0.08 c$ & H \\
\hline & $0.53 \pm 0.04 c$ & $2.43 \pm 0.01^{b}$ & $12.80 \pm 0.04^{c}$ & $0.08 \pm 0.01^{\mathrm{ab}}$ & $3.47 \pm 0.03^{a}$ & $0.18 \pm 0.02^{\mathrm{a}}$ & $19.47 \pm 0.10^{c}$ & Can \\
\hline & $0.72 \pm 0.11^{b}$ & $2.50 \pm 0.14^{b}$ & $12.89 \pm 0.52^{\mathrm{bc}}$ & $0.07 \pm 0.01 \mathrm{ab}$ & $3.30 \pm 0.04 a$ & $0.20 \pm 0.00^{a}$ & $19.68 \pm 0.30^{b c}$ & SB \\
\hline & $0.96 \pm 0.07 \mathrm{a}$ & $2.70 \pm 0.18^{b}$ & $13.19 \pm 0.27 \mathrm{bc}$ & $0.07 \pm 0.01 \mathrm{ab}$ & $2.68 \pm 0.15^{c}$ & $0.21 \pm 0.02^{\mathrm{a}}$ & $19.79 \pm 0.21 \mathrm{bc}$ & Corn \\
\hline & $0.40 \pm 0.03^{\mathrm{cd}}$ & $2.62 \pm 0.04^{b}$ & $13.48 \pm 0.13^{b}$ & $0.09 \pm 0.01^{a}$ & $3.39 \pm 0.04 a$ & $0.18 \pm 0.03^{a}$ & $20.15 \pm 0.27 b$ & 0 \\
\hline \multirow{7}{*}{6} & $0.42 \pm 0.00^{\mathrm{bc}}$ & $2.52 \pm 0.15^{b c}$ & $15.43 \pm 0.36^{a}$ & $0.08 \pm 0.01^{a}$ & $3.59 \pm 0.25^{a}$ & $0.17 \pm 0.00^{b}$ & $22.19 \pm 0.45^{\mathrm{a}}$ & C \\
\hline & $0.37 \pm 0.02^{\mathrm{bc}}$ & $2.55 \pm 0.12^{\mathrm{bc}}$ & $13.12 \pm 0.11^{d}$ & $0.08 \pm 0.00 \mathrm{a}$ & $3.49 \pm 0.02^{\mathrm{ab}}$ & $0.17 \pm 0.00^{b}$ & $19.76 \pm 0.23^{c}$ & s \\
\hline & $0.37 \pm 0.03^{b c}$ & $2.41 \pm 0.09 c$ & $13.71 \pm 0.03^{b c}$ & $0.07 \pm 0.00 \mathrm{a}$ & $3.32 \pm 0.08 \mathrm{abc}$ & $0.17 \pm 0.01^{b}$ & $20.04 \pm 0.06^{b c}$ & H \\
\hline & $0.32 \pm 0.04^{c}$ & $2.85 \pm 0.10^{a}$ & $13.14 \pm 0.18^{d}$ & $0.07 \pm 0.01^{a}$ & $3.52 \pm 0.06^{a b}$ & $0.20 \pm 0.00^{\mathrm{a}}$ & $20.10 \pm 0.40^{\mathrm{bc}}$ & Can \\
\hline & $0.76 \pm 0.06^{a}$ & $2.60 \pm 0.03 \mathrm{abc}$ & $13.10 \pm 0.18^{d}$ & $0.07 \pm 0.01^{a}$ & $3.03 \pm 0.10^{c}$ & $0.16 \pm 0.00^{b}$ & $19.72 \pm 0.15^{c}$ & SB \\
\hline & $0.47 \pm 0.04^{b}$ & $2.54 \pm 0.13^{\mathrm{bc}}$ & $13.43 \pm 0.04^{\mathrm{cd}}$ & $0.09 \pm 0.01^{a}$ & $3.26 \pm 0.08^{b c}$ & $0.18 \pm 0.02^{b}$ & $19.96 \pm 0.01^{c}$ & Corn \\
\hline & $0.47 \pm 0.06^{b}$ & $2.76 \pm 0.08^{\mathrm{ab}}$ & $14.04 \pm 0.15^{b}$ & $0.09 \pm 0.02^{\mathrm{a}}$ & $3.09 \pm 0.08 c$ & $0.17 \pm 0.01^{b}$ & $20.60 \pm 0.03^{b}$ & 0 \\
\hline \multirow{7}{*}{8} & $0.55 \pm 0.03^{b}$ & $2.69 \pm 0.12^{b}$ & $15.92 \pm 1.17^{a}$ & $0.08 \pm 0.01^{b}$ & $3.10 \pm 0.09 c$ & $0.17 \pm 0.01^{a}$ & $22.49 \pm 1.22^{\mathrm{a}}$ & C \\
\hline & $0.45 \pm 0.08^{b c}$ & $3.03 \pm 0.07^{a}$ & $13.54 \pm 0.27^{\mathrm{cd}}$ & $0.09 \pm 0.01 \mathrm{ab}$ & $3.14 \pm 0.06^{c}$ & $0.18 \pm 0.01^{\mathrm{ab}}$ & $20.42 \pm 0.22^{c}$ & s \\
\hline & $0.42 \pm 0.04 \mathrm{bc}$ & $2.58 \pm 0.07^{b}$ & $14.59 \pm 0.48^{\mathrm{bcd}}$ & $0.08 \pm 0.01^{b}$ & $2.92 \pm 0.03^{\mathrm{cd}}$ & $0.17 \pm 0.01^{b}$ & $20.75 \pm 0.40^{c}$ & H \\
\hline & $0.37 \pm 0.06^{c d}$ & $3.08 \pm 0.13^{a}$ & $14.83 \pm 0.13^{\mathrm{ab}}$ & $0.10 \pm 0.01^{a}$ & $3.64 \pm 0.06^{a}$ & $0.20 \pm 0.01^{\mathrm{ab}}$ & $22.20 \pm 0.13^{\mathrm{ab}}$ & Can \\
\hline & $0.69 \pm 0.04^{a}$ & $2.65 \pm 0.04^{b}$ & $13.43 \pm 0.11^{d}$ & $0.08 \pm 0.00^{\mathrm{ab}}$ & $3.08 \pm 0.11 c$ & $0.17 \pm 0.01^{b}$ & $20.09 \pm 0.22^{c}$ & SB \\
\hline & $0.26 \pm 0.07^{d}$ & $3.01 \pm 0.08^{a}$ & $14.11 \pm 0.13^{\mathrm{bcd}}$ & $0.09 \pm 0.01 \mathrm{ab}$ & $3.39 \pm 0.18^{b}$ & $0.22 \pm 0.04^{a}$ & $21.06 \pm 0.35 \mathrm{bc}$ & Corn \\
\hline & $0.43 \pm 0.05^{b c}$ & $2.78 \pm 0.06^{b}$ & $14.73 \pm 0.14 \mathrm{abc}$ & $0.08 \pm 0.00^{\mathrm{ab}}$ & $2.82 \pm 0.08^{d}$ & $0.18 \pm 0.02^{a b}$ & $21.01 \pm 0.15 b c$ & 0 \\
\hline \multirow{7}{*}{10} & $0.82 \pm 0.01^{a}$ & $3.10 \pm 0.05^{\mathrm{ab}}$ & $15.93 \pm 0.06^{a}$ & $0.10 \pm 0.02^{\mathrm{a}}$ & $2.51 \pm 0.46^{b}$ & $0.24 \pm 0.01^{a}$ & $22.68 \pm 0.36^{a}$ & C \\
\hline & $0.64 \pm 0.06^{\mathrm{cd}}$ & $3.18 \pm 0.04 \mathrm{a}$ & $14.86 \pm 0.09 \mathrm{bc}$ & $0.07 \pm 0.01^{a}$ & $3.34 \pm 0.04^{a}$ & $0.20 \pm 0.01 \mathrm{ab}$ & $22.27 \pm 0.13^{\mathrm{ab}}$ & S \\
\hline & $0.58 \pm 0.03^{\mathrm{cd}}$ & $3.01 \pm 0.09 \mathrm{ab}$ & $14.88 \pm 0.14^{b c}$ & $0.08 \pm 0.01^{a}$ & $2.87 \pm 0.13^{\mathrm{ab}}$ & $0.22 \pm 0.02^{\mathrm{ab}}$ & $21.62 \pm 0.16^{b c}$ & H \\
\hline & $0.66 \pm 0.03^{b c}$ & $3.23 \pm 0.11^{\mathrm{a}}$ & $15.12 \pm 0.17^{b}$ & $0.09 \pm 0.01^{a}$ & $3.12 \pm 0.01^{a}$ & $0.18 \pm 0.03^{b}$ & $22.40 \pm 0.01^{\mathrm{a}}$ & Can \\
\hline & $0.73 \pm 0.04^{b}$ & $3.02 \pm 0.18^{\mathrm{ab}}$ & $14.17 \pm 0.23^{d}$ & $0.09 \pm 0.02^{\mathrm{a}}$ & $3.05 \pm 0.11^{\mathrm{a}}$ & $0.20 \pm 0.01^{\mathrm{ab}}$ & $21.25 \pm 0.59 c$ & SB \\
\hline & $0.57 \pm 0.03^{\mathrm{de}}$ & $2.99 \pm 0.04^{\mathrm{ab}}$ & $15.82 \pm 0.11^{a}$ & $0.09 \pm 0.02^{\mathrm{a}}$ & $3.24 \pm 0.10^{a}$ & $0.20 \pm 0.01^{\mathrm{ab}}$ & $22.89 \pm 0.24 \mathrm{a}$ & Corn \\
\hline & $0.49 \pm 0.01 \mathrm{e}$ & $2.86 \pm 0.13^{b}$ & $14.70 \pm 0.03^{c}$ & $0.07 \pm 0.01^{\mathrm{a}}$ & $2.86 \pm 0.11^{\mathrm{ab}}$ & $0.21 \pm 0.03^{\mathrm{ab}}$ & $21.18 \pm 0.21^{c}$ & 0 \\
\hline \multirow{7}{*}{12} & $0.52 \pm 0.05^{c}$ & $3.68 \pm 0.11^{a}$ & $16.50 \pm 0.10^{a}$ & $0.08 \pm 0.01^{a}$ & $3.10 \pm 0.21 \mathrm{ab}$ & $0.19 \pm 0.02^{b}$ & $24.05 \pm 0.13^{a}$ & C \\
\hline & $0.55 \pm 0.01^{c}$ & $3.08 \pm 0.06^{d}$ & $15.92 \pm 0.09 b$ & $0.07 \pm 0.01^{a}$ & $2.95 \pm 0.08^{b}$ & $0.22 \pm 0.02^{\mathrm{ab}}$ & $22.78 \pm 0.17 c$ & s \\
\hline & $0.78 \pm 0.10^{\mathrm{ab}}$ & $3.51 \pm 0.03^{\mathrm{ab}}$ & $15.69 \pm 0.08^{b}$ & $0.07 \pm 0.00^{a}$ & $3.11 \pm 0.14^{\mathrm{ab}}$ & $0.20 \pm 0.01^{b}$ & $23.35 \pm 0.00^{\mathrm{b}}$ & H \\
\hline & $0.89 \pm 0.04^{a}$ & $3.37 \pm 0.12^{\mathrm{bc}}$ & $15.79 \pm 0.19 b$ & $0.08 \pm 0.01^{a}$ & $3.08 \pm 0.11 \mathrm{ab}$ & $0.25 \pm 0.02^{\mathrm{a}}$ & $23.43 \pm 0.35^{b}$ & Can \\
\hline & $0.90 \pm 0.01^{\mathrm{a}}$ & $3.41 \pm 0.20^{\mathrm{ab}}$ & $15.16 \pm 0.11 \mathrm{c}$ & $0.08 \pm 0.02^{\mathrm{a}}$ & $3.48 \pm 0.28^{a}$ & $0.19 \pm 0.00^{\mathrm{b}}$ & $23.21 \pm 0.20^{b}$ & SB \\
\hline & $0.67 \pm 0.04^{b}$ & $3.11 \pm 0.13^{\mathrm{cd}}$ & $15.94 \pm 0.01^{b}$ & $0.08 \pm 0.00^{a}$ & $3.29 \pm 0.20^{\mathrm{ab}}$ & $0.22 \pm 0.02^{\mathrm{ab}}$ & $23.31 \pm 0.12^{b}$ & Corn \\
\hline & $0.56 \pm 0.04 c$ & $3.08 \pm 0.03^{d}$ & $15.08 \pm 0.06^{c}$ & $0.08 \pm 0.01^{a}$ & $3.03 \pm 0.07^{b}$ & $0.18 \pm 0.03^{b}$ & $21.97 \pm 0.08^{d}$ & 0 \\
\hline
\end{tabular}

Means sharing the same letter in the same row (a-e) are not significantly different ( $p>0.05)$ using Duncan's multiple range test. C: Control, S: sunflower, $\mathrm{H}$ : hazelnut, Can: Canola, SB: Soybean, Corn: Corn, O: Olive. 
Table 2. Changes in MUFA (\%) content of sea bass treated with/without nanoemulsions during storage period

\begin{tabular}{|c|c|c|c|c|c|c|c|c|}
\hline Days & C14:1 & C16:1 & C17:1 & C18:1n9 & C20:1 & C22:1n9 & MUFA & Groups \\
\hline 0 & $0.08 \pm 0.00$ & $4.71 \pm 0.10$ & $0.10 \pm 0.00$ & $29.95 \pm 0.47$ & $0.83 \pm 0.08$ & $0.22 \pm 0.04$ & $35.88 \pm 0.25$ & $C$ \\
\hline \multirow{7}{*}{2} & $0.09 \pm 0.01^{a}$ & $4.20 \pm 0.11^{\mathrm{a}}$ & $0.11 \pm 0.00^{a}$ & $28.30 \pm 0.30^{b}$ & $1.03 \pm 0.00^{a}$ & $0.24 \pm 0.03^{a}$ & $33.96 \pm 0.37 c$ & C \\
\hline & $0.11 \pm 0.02^{\mathrm{a}}$ & $4.79 \pm 0.07^{a}$ & $0.11 \pm 0.01^{a}$ & $30.18 \pm 0.11^{a}$ & $0.88 \pm 0.10^{\mathrm{abc}}$ & $0.24 \pm 0.04^{a}$ & $36.30 \pm 0.35^{a}$ & $S$ \\
\hline & $0.10 \pm 0.01 \mathrm{a}$ & $4.76 \pm 0.04 a$ & $0.10 \pm 0.01 \mathrm{a}$ & $30.27 \pm 0.08 \mathrm{a}$ & $0.74 \pm 0.03^{c}$ & $0.21 \pm 0.03^{a}$ & $36.17 \pm 0.06 \mathrm{ab}$ & H \\
\hline & $0.07 \pm 0.04^{a}$ & $4.61 \pm 0.03^{a}$ & $0.10 \pm 0.01^{a}$ & $30.12 \pm 0.11^{a}$ & $1.04 \pm 0.14 a$ & $0.22 \pm 0.02^{\mathrm{a}}$ & $36.15 \pm 0.25 \mathrm{ab}$ & Can \\
\hline & $0.09 \pm 0.01^{a}$ & $4.70 \pm 0.09 \mathrm{a}$ & $0.11 \pm 0.01 \mathrm{a}$ & $30.15 \pm 0.07 a$ & $0.81 \pm 0.13^{b c}$ & $0.12 \pm 0.02^{b}$ & $35.97 \pm 0.21 \mathrm{ab}$ & SB \\
\hline & $0.08 \pm 0.01^{a}$ & $4.25 \pm 0.61^{\mathrm{a}}$ & $0.10 \pm 0.01^{a}$ & $30.13 \pm 0.52^{\mathrm{a}}$ & $0.91 \pm 0.06^{\mathrm{abc}}$ & $0.24 \pm 0.01^{\mathrm{a}}$ & $35.70 \pm 0.00^{\mathrm{b}}$ & Corn \\
\hline & $0.07 \pm 0.03^{a}$ & $4.32 \pm 0.48^{a}$ & $0.09 \pm 0.01^{a}$ & $30.45 \pm 0.40^{a}$ & $0.96 \pm 0.00^{\mathrm{ab}}$ & $0.27 \pm 0.03^{a}$ & $36.16 \pm 0.01^{\mathrm{ab}}$ & 0 \\
\hline \multirow{7}{*}{4} & $0.11 \pm 0.01^{a}$ & $4.04 \pm 0.01 \mathrm{e}$ & $0.10 \pm 0.04 a$ & $28.68 \pm 0.44 c$ & $0.80 \pm 0.02^{\mathrm{cd}}$ & $0.28 \pm 0.01^{a}$ & $33.99 \pm 0.38^{d}$ & C \\
\hline & $0.08 \pm 0.01^{\mathrm{ab}}$ & $4.61 \pm 0.05 b^{c}$ & $0.09 \pm 0.00^{a}$ & $29.24 \pm 0.16^{b}$ & $0.86 \pm 0.06^{b c}$ & $0.21 \pm 0.06^{\mathrm{ab}}$ & $35.08 \pm 0.09 b$ & $S$ \\
\hline & $0.09 \pm 0.00^{\mathrm{ab}}$ & $4.75 \pm 0.15^{\mathrm{ab}}$ & $0.10 \pm 0.00 \mathrm{a}$ & $30.17 \pm 0.04^{a}$ & $0.73 \pm 0.01^{d}$ & $0.26 \pm 0.01^{\mathrm{ab}}$ & $36.09 \pm 0.13^{a}$ & H \\
\hline & $0.08 \pm 0.00^{\mathrm{ab}}$ & $4.52 \pm 0.04 c$ & $0.10 \pm 0.01^{a}$ & $29.94 \pm 0.09 \mathrm{a}$ & $0.88 \pm 0.01 \mathrm{bc}$ & $0.18 \pm 0.04^{b}$ & $35.69 \pm 0.07^{a}$ & Can \\
\hline & $0.07 \pm 0.01^{b}$ & $4.69 \pm 0.01 \mathrm{bc}$ & $0.10 \pm 0.01^{a}$ & $30.04 \pm 0.02^{a}$ & $0.83 \pm 0.04 \mathrm{bc}$ & $0.18 \pm 0.01^{b}$ & $35.89 \pm 0.01^{a}$ & SB \\
\hline & $0.07 \pm 0.01^{b}$ & $4.27 \pm 0.08^{d}$ & $0.09 \pm 0.01^{a}$ & $28.73 \pm 0.25^{c}$ & $1.17 \pm 0.01^{a}$ & $0.25 \pm 0.05^{\mathrm{ab}}$ & $34.56 \pm 0.24 c$ & Corn \\
\hline & $0.10 \pm 0.03 \mathrm{a}$ & $4.92 \pm 0.12^{\mathrm{a}}$ & $0.12 \pm 0.02^{\mathrm{a}}$ & $29.70 \pm 0.07 \mathrm{ab}$ & $0.92 \pm 0.06^{b}$ & $0.25 \pm 0.01 \mathrm{ab}$ & $36.00 \pm 0.15^{a}$ & 0 \\
\hline \multirow{7}{*}{6} & $0.07 \pm 0.01^{a}$ & $3.88 \pm 0.11^{d}$ & $0.10 \pm 0.01^{a}$ & $28.66 \pm 0.41 \mathrm{de}$ & $0.85 \pm 0.07 a$ & $0.18 \pm 0.01^{b}$ & $33.74 \pm 0.57 c$ & $C$ \\
\hline & $0.06 \pm 0.06^{a}$ & $4.33 \pm 0.08^{b}$ & $0.11 \pm 0.01^{a}$ & $29.04 \pm 0.12^{\mathrm{cd}}$ & $0.79 \pm 0.02^{\mathrm{ab}}$ & $0.21 \pm 0.05^{\mathrm{ab}}$ & $34.51 \pm 0.23^{b}$ & $S$ \\
\hline & $0.07 \pm 0.01^{\mathrm{a}}$ & $4.31 \pm 0.08^{b}$ & $0.09 \pm 0.00^{a}$ & $29.80 \pm 0.08^{a}$ & $0.75 \pm 0.02^{b}$ & $0.27 \pm 0.03^{a}$ & $35.28 \pm 0.04 a$ & H \\
\hline & $0.08 \pm 0.03^{a}$ & $4.83 \pm 0.07^{a}$ & $0.10 \pm 0.02^{\mathrm{a}}$ & $29.30 \pm 0.07 b c$ & $0.83 \pm 0.02^{\mathrm{ab}}$ & $0.28 \pm 0.04^{a}$ & $35.41 \pm 0.08^{a}$ & Can \\
\hline & $0.07 \pm 0.03^{a}$ & $4.06 \pm 0.07^{c}$ & $0.10 \pm 0.00^{a}$ & $29.96 \pm 0.09^{a}$ & $0.80 \pm 0.04^{\mathrm{ab}}$ & $0.26 \pm 0.01^{\mathrm{a}}$ & $35.24 \pm 0.00^{a}$ & SB \\
\hline & $0.08 \pm 0.01^{a}$ & $4.32 \pm 0.07^{b}$ & $0.10 \pm 0.00^{a}$ & $28.39 \pm 0.22^{\mathrm{e}}$ & $0.76 \pm 0.04 \mathrm{ab}$ & $0.18 \pm 0.00^{b}$ & $33.83 \pm 0.18 c$ & Corn \\
\hline & $0.08 \pm 0.02^{\mathrm{a}}$ & $4.20 \pm 0.06^{\mathrm{bc}}$ & $0.11 \pm 0.01^{\mathrm{a}}$ & $29.58 \pm 0.07$ ab & $0.81 \pm 0.01^{\mathrm{ab}}$ & $0.26 \pm 0.02^{\mathrm{a}}$ & $35.02 \pm 0.01^{\mathrm{ab}}$ & 0 \\
\hline \multirow{7}{*}{8} & $0.08 \pm 0.00^{b}$ & $3.81 \pm 0.23^{d}$ & $0.10 \pm 0.01^{b}$ & $28.39 \pm 0.28^{\mathrm{cd}}$ & $0.71 \pm 0.01^{c}$ & $0.27 \pm 0.01 \mathrm{ab}$ & $33.35 \pm 0.05^{d}$ & $C$ \\
\hline & $0.08 \pm 0.00^{b}$ & $4.44 \pm 0.09 b$ & $0.11 \pm 0.01 \mathrm{ab}$ & $28.49 \pm 0.11 \mathrm{~cd}$ & $0.89 \pm 0.11 \mathrm{ab}$ & $0.20 \pm 0.05^{c}$ & $34.20 \pm 0.19 c$ & S \\
\hline & $0.09 \pm 0.00^{\mathrm{ab}}$ & $3.93 \pm 0.03^{\mathrm{cd}}$ & $0.10 \pm 0.00^{b}$ & $29.41 \pm 0.13 \mathrm{ab}$ & $0.80 \pm 0.04 b c$ & $0.26 \pm 0.02^{\mathrm{abc}}$ & $34.58 \pm 0.11 \mathrm{bc}$ & H \\
\hline & $0.08 \pm 0.03^{b}$ & $4.85 \pm 0.14^{a}$ & $0.13 \pm 0.00 \mathrm{a}$ & $28.78 \pm 0.40^{c}$ & $0.93 \pm 0.03^{a}$ & $0.30 \pm 0.01^{\mathrm{a}}$ & $35.07 \pm 0.56 \mathrm{ab}$ & Can \\
\hline & $0.09 \pm 0.00^{\mathrm{ab}}$ & $4.19 \pm 0.01^{b c}$ & $0.10 \pm 0.00^{b}$ & $29.81 \pm 0.08^{a}$ & $0.79 \pm 0.01 \mathrm{bc}$ & $0.19 \pm 0.01^{c}$ & $35.16 \pm 0.07^{a}$ & SB \\
\hline & $0.10 \pm 0.01 \mathrm{ab}$ & $4.12 \pm 0.06^{c}$ & $0.11 \pm 0.01 \mathrm{ab}$ & $28.16 \pm 0.06^{d}$ & $0.87 \pm 0.06^{\mathrm{ab}}$ & $0.27 \pm 0.03^{\mathrm{ab}}$ & $33.62 \pm 0.01^{\mathrm{d}}$ & Corn \\
\hline & $0.11 \pm 0.00 \mathrm{a}$ & $4.11 \pm 0.11 \mathrm{c}$ & $0.12 \pm 0.01 \mathrm{ab}$ & $29.28 \pm 0.16^{b}$ & $0.73 \pm 0.03 c$ & $0.23 \pm 0.04 \mathrm{bc}$ & $34.57 \pm 0.02^{\mathrm{bc}}$ & 0 \\
\hline \multirow{7}{*}{10} & $0.09 \pm 0.00^{a}$ & $3.89 \pm 0.06^{c}$ & $0.12 \pm 0.01^{a}$ & $23.88 \pm 0.54^{b}$ & $0.87 \pm 0.01^{\mathrm{ab}}$ & $0.26 \pm 0.02^{b c}$ & $29.10 \pm 0.46^{c}$ & $C$ \\
\hline & $0.07 \pm 0.00^{a}$ & $4.68 \pm 0.06^{a}$ & $0.09 \pm 0.00^{a}$ & $27.05 \pm 0.14^{a}$ & $0.77 \pm 0.01^{c}$ & $0.18 \pm 0.01^{d}$ & $32.84 \pm 0.21 \mathrm{ab}$ & S \\
\hline & $0.09 \pm 0.01^{a}$ & $3.76 \pm 0.09 c$ & $0.10 \pm 0.01^{a}$ & $27.62 \pm 0.09 \mathrm{a}$ & $0.87 \pm 0.08^{\mathrm{ab}}$ & $0.21 \pm 0.01 \mathrm{~cd}$ & $32.64 \pm 0.12^{\mathrm{ab}}$ & H \\
\hline & $0.09 \pm 0.00^{a}$ & $4.13 \pm 0.05^{b}$ & $0.12 \pm 0.02^{\mathrm{a}}$ & $27.52 \pm 0.30 \mathrm{a}$ & $0.81 \pm 0.04 \mathrm{bc}$ & $0.27 \pm 0.02^{b}$ & $32.92 \pm 0.27 \mathrm{ab}$ & Can \\
\hline & $0.08 \pm 0.00^{a}$ & $4.27 \pm 0.11^{b}$ & $0.11 \pm 0.00 \mathrm{a}$ & $27.02 \pm 0.25^{a}$ & $0.89 \pm 0.00^{\mathrm{ab}}$ & $0.12 \pm 0.01 \mathrm{e}$ & $32.49 \pm 0.15^{b}$ & SB \\
\hline & $0.08 \pm 0.03^{a}$ & $4.24 \pm 0.04^{b}$ & $0.11 \pm 0.01^{a}$ & $27.52 \pm 0.24^{a}$ & $0.91 \pm 0.01^{a}$ & $0.34 \pm 0.01^{\mathrm{a}}$ & $33.19 \pm 0.21^{\mathrm{a}}$ & Corn \\
\hline & $0.08 \pm 0.01 \mathrm{a}$ & $4.26 \pm 0.09 \mathrm{~b}$ & $0.09 \pm 0.02^{\mathrm{a}}$ & $27.11 \pm 0.13^{\mathrm{a}}$ & $0.74 \pm 0.01 \mathrm{c}$ & $0.22 \pm 0.04 \mathrm{~cd}$ & $32.48 \pm 0.02^{b}$ & 0 \\
\hline \multirow{7}{*}{12} & $0.06 \pm 0.01^{\mathrm{ab}}$ & $3.72 \pm 0.09 \mathrm{ab}$ & $0.10 \pm 0.01^{a}$ & $23.82 \pm 0.44^{d}$ & $0.83 \pm 0.02^{\mathrm{ab}}$ & $0.29 \pm 0.01^{\mathrm{ab}}$ & $28.80 \pm 0.30 c$ & $C$ \\
\hline & $0.09 \pm 0.03^{a}$ & $3.75 \pm 0.11^{\mathrm{ab}}$ & $0.10 \pm 0.01^{a}$ & $25.04 \pm 0.11 \mathrm{abc}$ & $0.89 \pm 0.04 a$ & $0.29 \pm 0.03^{a}$ & $30.15 \pm 0.10^{b}$ & S \\
\hline & $0.09 \pm 0.01 \mathrm{ab}$ & $3.60 \pm 0.01^{b c}$ & $0.09 \pm 0.00^{a}$ & $24.44 \pm 0.16^{\mathrm{cd}}$ & $0.68 \pm 0.01^{b}$ & $0.22 \pm 0.00^{c}$ & $29.11 \pm 0.14 c$ & H \\
\hline & $0.09 \pm 0.00^{a}$ & $3.76 \pm 0.09 a b$ & $0.10 \pm 0.01^{a}$ & $24.96 \pm 0.01 \mathrm{bc}$ & $0.76 \pm 0.06^{\mathrm{ab}}$ & $0.09 \pm 0.03^{d}$ & $29.75 \pm 0.18^{b}$ & Can \\
\hline & $0.06 \pm 0.01 b$ & $3.88 \pm 0.02^{a}$ & $0.11 \pm 0.03^{a}$ & $25.75 \pm 0.54 a$ & $0.72 \pm 0.10^{\mathrm{ab}}$ & $0.25 \pm 0.01 \mathrm{abc}$ & $30.76 \pm 0.42^{\mathrm{a}}$ & SB \\
\hline & $0.09 \pm 0.00^{a}$ & $3.42 \pm 0.12^{c}$ & $0.11 \pm 0.01^{a}$ & $25.17 \pm 0.13 \mathrm{ab}$ & $0.82 \pm 0.13^{\mathrm{a} b}$ & $0.28 \pm 0.04 \mathrm{abc}$ & $29.87 \pm 0.17^{b}$ & Corn \\
\hline & $0.09 \pm 0.01 a$ & $3.88 \pm 0.12^{d}$ & $0.12 \pm 0.02^{\mathrm{a}}$ & $25.74 \pm 0.14 \mathrm{a}$ & $0.76 \pm 0.06 \mathrm{ab}$ & $0.21 \pm 0.03^{b c}$ & $30.61 \pm 0.22^{\mathrm{a}}$ & 0 \\
\hline
\end{tabular}

Means sharing the same letter in the same row $(a-e)$ are not significantly different $(p>0.05)$ using Duncan's multiple range test. C: Control, S: sunflower, H: hazelnut, Can: Canola, SB: Soybean, Corn: Corn, O: Olive. 
The changes in PUFA content of seabass fillets treated with nanoemulsions and stored in cold storage are given in Table 3. The initial PUFA value was determined as $29.25 \%$. This value decreased over the storage period and at the end of the storage period it was determined as $20.94 \%, 24.30 \%, 23.70 \%, 24.46 \%$, $26.41 \%, 23.78 \%, 24.48 \%$ for the control, sunflower, hazelnut, canola, soybean, corn and olive oil groups respectively. Similarly, Ozogul et al. (2017) reported that the PUFA amount of sea bass decreased with storage time. While corn oil nanoemulsion group was found to have the highest PUFA value $(29.83 \%)$ on the second day of storage, the control group had the lowest PUFA value (20.94\%) on day 12.

The main fatty acids among PUFA in all groups were linoleic acid, eicosapentaenoic acid $\left(\mathrm{C}_{20: 5} \mathrm{n} 3\right)$ and

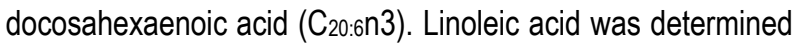
as $14.52 \%$ at the beginning of the storage period, and this value decreased throughout the storage period. Statistically significant differences were observed between groups during the storage period. At the end of the storage period, hazelnut oil group had the lowest linoleic acid value $(13.36 \%)$ and soybean oil group had the highest linoleic acid value (14.87\%). Yazgan (2013) reported the linoleic acid levels of nanoemulsion treatment groups in sea bream as $10.13 \%$ and $11.28 \%$ at the beginning and the end of the storage period respectively. Lenas et al. (2011) reported the linoleic acid level of wild caught sea bass as $6.39 \%$ and cultured sea bass as $18.05 \%$. This difference in linoleic acid levels was thought to arise from the contents of feed used in aquaculture.

Table 3. Changes in PUFA (\%) content of sea bass treated with/without nanoemulsions during storage period

\begin{tabular}{|c|c|c|c|c|c|c|c|c|c|c|}
\hline Days & C18:2n6 & C18:3n3 & C20:2 & C20:3n6 & C20:5n3 & C20:4n6 & C22.2 & C22:6n3 & PUFA & Groups \\
\hline 0 & $14.52 \pm 0.42$ & $0.56 \pm 0.01$ & $19 \pm 0.00$ & $0.42 \pm 0.00$ & $4.21 \pm 0.08$ & $1.05 \pm 0.07$ & $0.23 \pm 0.04$ & $8.09 \pm 0.19$ & .35 & $C$ \\
\hline \multirow{7}{*}{2} & $14.54 \pm 0.09_{c d}$ & $0.54 \pm 0.04 \mathrm{ab}$ & $0.19 \pm 0.00^{a}$ & $0.44 \pm 0.00^{a}$ & $4.10 \pm 0.03^{c}$ & $0.47 \pm 0.04^{c}$ & $0.28 \pm 0.00^{a}$ & $6.42 \pm 0.56^{b}$ & $26.97 \pm 0.52^{c}$ & C \\
\hline & $14.20 \pm 0.14^{\mathrm{d}}$ & $0.55 \pm 0.04 a$ & $0.20 \pm 0.05^{a}$ & $0.47 \pm 0.07^{a}$ & $4.55 \pm 0.14^{\mathrm{ab}}$ & $1.35 \pm 0.20^{a}$ & $0.22 \pm 0.04 \mathrm{bc}$ & $7.88 \pm 0.23^{a}$ & $29.41 \pm 0.60^{\mathrm{ab}}$ & S \\
\hline & $14.73 \pm 0.15^{b c}$ & $0.52 \pm 0.01 \mathrm{ab}$ & $0.19 \pm 0.01^{a}$ & $0.41 \pm 0.00^{a}$ & $4.60 \pm 0.01^{\mathrm{ab}}$ & $0.23 \pm 0.04 c$ & $0.18 \pm 0.01^{c}$ & $7.48 \pm 0.25^{a}$ & $28.31 \pm 0.33^{b}$ & $\mathrm{H}$ \\
\hline & $15.08 \pm 0.25^{\mathrm{ab}}$ & $0.46 \pm 0.04 b$ & $0.20 \pm 0.02^{a}$ & $0.44 \pm 0.04 a$ & $4.40 \pm 0.07^{b}$ & $0.30 \pm 0.08^{c}$ & $0.25 \pm 0.02^{\mathrm{ab}}$ & $7.97 \pm 0.22^{\mathrm{a}}$ & $29.08 \pm 0.11 \mathrm{ab}$ & Can \\
\hline & $15.44 \pm 0.02^{\mathrm{a}}$ & $0.56 \pm 0.04 a$ & $0.19 \pm 0.01^{a}$ & $0.47 \pm 0.07 \mathrm{a}$ & $4.42 \pm 0.19^{b}$ & $0.72 \pm 0.11^{b}$ & $0.20 \pm 0.04^{b c}$ & $7.68 \pm 0.25^{a}$ & $29.66 \pm 0.42^{a}$ & SB \\
\hline & $15.15 \pm 0.06 \mathrm{ab}$ & $0.50 \pm 0.03 \mathrm{ab}$ & $0.19 \pm 0.01 \mathrm{a}$ & $0.42 \pm 0.02^{a}$ & $4.73 \pm 0.08 \mathrm{a}$ & $1.25 \pm 0.11 \mathrm{a}$ & $0.21 \pm 0.01 \mathrm{bc}$ & $7.41 \pm 0.22^{a}$ & $29.83 \pm 0.54 a$ & Corn \\
\hline & $14.70 \pm 0.39 \mathrm{bc}$ & $0.50 \pm 0.01^{\mathrm{ab}}$ & $0.19 \pm 0.02^{a}$ & $0.42 \pm 0.05^{\mathrm{a}}$ & $4.57 \pm 0.04^{\mathrm{ab}}$ & $0.26 \pm 0.04 c$ & $0.23 \pm 0.03^{\mathrm{abc}}$ & $7.38 \pm 0.21 \mathrm{a}$ & $28.22 \pm 0.70^{b}$ & 0 \\
\hline \multirow{7}{*}{4} & $14.26 \pm 0.88^{b}$ & $0.54 \pm 0.08 \mathrm{ab}$ & $0.25 \pm 0.03^{a}$ & $0.50 \pm 0.05^{a}$ & $3.79 \pm 0.01^{b}$ & $0.86 \pm 0.06^{b}$ & $0.22 \pm 0.01^{\mathrm{ab}}$ & $6.19 \pm 0.92^{b}$ & $26.59 \pm 1.94^{b}$ & C \\
\hline & $14.18 \pm 0.04^{b}$ & $0.52 \pm 0.01^{\mathrm{ab}}$ & $0.26 \pm 0.05^{a}$ & $0.46 \pm 0.02^{a}$ & $4.46 \pm 0.15^{a}$ & $1.39 \pm 0.08^{a}$ & $0.20 \pm 0.00^{\mathrm{bc}}$ & $6.80 \pm 0.24 \mathrm{ab}$ & $28.24 \pm 0.21^{a}$ & $S$ \\
\hline & $14.37 \pm 0.18^{b}$ & $0.50 \pm 0.03^{\mathrm{a} a}$ & $0.20 \pm 0.01^{a}$ & $0.44 \pm 0.01^{a}$ & $4.21 \pm 0.07^{a}$ & $0.37 \pm 0.05^{c}$ & $0.17 \pm 0.01 c$ & $7.06 \pm 0.06^{\mathrm{ab}}$ & $27.31 \pm 0.12^{\mathrm{ab}}$ & $\mathrm{H}$ \\
\hline & $14.79 \pm 0.10^{\mathrm{ab}}$ & $0.48 \pm 0.04 c$ & $0.19 \pm 0.00^{a}$ & $0.41 \pm 0.01^{a}$ & $4.19 \pm 0.10^{\mathrm{a}}$ & $0.33 \pm 0.02^{c}$ & $0.20 \pm 0.01^{b c}$ & $6.59 \pm 0.14 \mathrm{ab}$ & $27.17 \pm 0.20^{\mathrm{ab}}$ & Can \\
\hline & $15.51 \pm 0.31^{\mathrm{a}}$ & $0.52 \pm$ & & & & & & $7.52 \pm 0.06^{a}$ & $92 \pm 0.06 \mathrm{ab}$ & SB \\
\hline & $14.15 \pm 0.21^{b}$ & $0.48 \pm 0.02^{c}$ & $0.23 \pm 0.01^{a}$ & $0.45 \pm 0.03^{a}$ & $4.37 \pm 0.20^{a}$ & $1.47 \pm 0.12^{\mathrm{a}}$ & $0.26 \pm 0.03^{a}$ & $6.49 \pm 0.10^{b}$ & $27.89 \pm 0.11 \mathrm{ab}$ & Corn \\
\hline & $14.51 \pm 0.21^{\mathrm{b}}$ & $0.59 \pm 0.04 a$ & $0.22 \pm 0.04 a$ & $0.49 \pm 0.07 \mathrm{a}$ & $4.18 \pm 0.07^{a}$ & $0.35 \pm 0.05^{c}$ & $0.22 \pm 0.04^{\mathrm{ab}}$ & $6.96 \pm 0.11 \mathrm{ab}$ & $27.50 \pm 0.06^{\mathrm{ab}}$ & 0 \\
\hline \multirow{7}{*}{6} & $14.28 \pm 0.37 \mathrm{~b}$ & $0.47 \pm 0.03^{a}$ & $0.20 \pm 0.00^{a}$ & $0.45 \pm 0.01^{a}$ & $3.39 \pm 0.12^{d}$ & $0.35 \pm 0.01^{\mathrm{a}}$ & $0.21 \pm 0.03^{a}$ & $5.56 \pm 0.11^{c}$ & $24.90 \pm 0.30^{c}$ & $C$ \\
\hline & $13.89 \pm 0.06^{b}$ & $0.53 \pm 0.01^{a}$ & $0.19 \pm 0.00^{a}$ & $0.43 \pm 0.01^{a}$ & $4.38 \pm 0.04^{a}$ & $0.52 \pm 0.02^{\mathrm{a}}$ & $0.18 \pm 0.01^{a}$ & $33 \pm 0.01^{b}$ & $26.44 \pm 0.09 \mathrm{~b}$ & $S$ \\
\hline & $14.17 \pm 0.04^{b}$ & $0.50 \pm 0.03^{a}$ & $0.19 \pm 0.00^{a}$ & $0.44 \pm 0.00^{a}$ & $4.07 \pm 0.07^{b}$ & $0.31 \pm 0.01^{\mathrm{a}}$ & $0.18 \pm 0.01^{a}$ & $6.87 \pm 0.07 \mathrm{a}$ & $26.73 \pm 0.06^{\mathrm{ab}}$ & H \\
\hline & $14.86 \pm 0.13^{a}$ & $0.38 \pm 0.21 \mathrm{a}$ & $0.21 \pm 0.02^{a}$ & $0.44 \pm 0.02^{\mathrm{a}}$ & $4.15 \pm 0.08^{b}$ & $0.28 \pm 0.01^{a}$ & $0.20 \pm 0.01^{a}$ & $6.43 \pm 0.04^{b}$ & $26.93 \pm 0.44^{\mathrm{ab}}$ & Can \\
\hline & $15.15 \pm 0.11 \mathrm{a}$ & $0.53 \pm 0.03 \mathrm{a}$ & $0.19 \pm 0.00^{a}$ & $0.44 \pm 0.01 \mathrm{a}$ & $3.83 \pm 0.15^{c}$ & $0.23 \pm 0.04 a$ & $0.19 \pm 0.01 \mathrm{a}$ & $6.60 \pm 0.28 \mathrm{ab}$ & $27.15 \pm 0.33 \mathrm{ab}$ & SB \\
\hline & $14.91 \pm 0.09 \mathrm{a}$ & $0.47 \pm 0.03 a$ & $0.19 \pm 0.01 \mathrm{a}$ & $0.43 \pm 0.01 \mathrm{a}$ & $4.20 \pm 0.07 \mathrm{ab}$ & $0.81 \pm 0.70^{a}$ & $0.18 \pm 0.00 \mathrm{a}$ & $6.31 \pm 0.06^{b}$ & $27.49 \pm 0.78^{a}$ & Corn \\
\hline & $14.22 \pm 0.04^{b}$ & $0.55 \pm 0.02^{\mathrm{a}}$ & $0.20 \pm 0.01^{\mathrm{a}}$ & $0.44 \pm 0.01^{\mathrm{a}}$ & $4.04 \pm 0.09 \mathrm{bc}$ & $0.30 \pm 0.03^{a}$ & $0.20 \pm 0.01^{\mathrm{a}}$ & $6.76 \pm 0.16^{a}$ & $26.69 \pm 0.21 \mathrm{ab}$ & 0 \\
\hline \multirow{7}{*}{8} & $13.76 \pm 0.41^{b}$ & $0.43 \pm 0.04 \mathrm{ab}$ & $0.20 \pm 0.01^{a}$ & $0.46 \pm 0.01 \mathrm{bc}$ & $3.18 \pm 0.01 \mathrm{~d}$ & $0.34 \pm 0.03 \mathrm{ab}$ & $0.19 \pm 0.01 b$ & $5.31 \pm 0.40^{d}$ & $23.86 \pm 0.86^{d}$ & $C$ \\
\hline & $13.80 \pm 0.07^{b}$ & $0.49 \pm 0.01^{\mathrm{ab}}$ & $0.22 \pm 0.03^{a}$ & $0.49 \pm 0.04^{\mathrm{ab}}$ & $4.24 \pm 0.08^{a}$ & $0.33 \pm 0.02^{\mathrm{ab}}$ & $0.20 \pm 0.05^{\mathrm{ab}}$ & $6.03 \pm 0.08 \mathrm{bc}$ & $25.79 \pm 0.01^{c}$ & $S$ \\
\hline & $14.12 \pm 0.08^{b}$ & $0.48 \pm 0.01^{\mathrm{ab}}$ & $0.19 \pm 0.00^{a}$ & $0.44 \pm 0.01^{c}$ & $3.94 \pm 0.06^{b c}$ & $0.27 \pm 0.03^{b}$ & $0.22 \pm 0.01^{\mathrm{ab}}$ & $6.73 \pm 0.05^{\mathrm{a}}$ & $26.36 \pm 0.14^{\mathrm{abc}}$ & H \\
\hline & $14.77 \pm 0.14^{a}$ & $0.35 \pm 0.14^{b}$ & $0.23 \pm 0.00^{a}$ & $0.52 \pm 0.01^{a}$ & $4.11 \pm 0.10^{\mathrm{ab}}$ & $0.37 \pm 0.05^{a}$ & $0.23 \pm 0.01^{a}$ & $6.19 \pm 0.09 \mathrm{bc}$ & $26.75 \pm 0.24 \mathrm{ab}$ & Can \\
\hline & $15.14 \pm 0.37^{\mathrm{a}}$ & $0.51 \pm 0.01^{a}$ & $0.20 \pm 0.01^{a}$ & $0.46 \pm 0.01^{\mathrm{bc}}$ & $3.79 \pm 0.09 c$ & $0.30 \pm 0.01 \mathrm{ab}$ & $0.19 \pm 0.01^{\mathrm{ab}}$ & $6.40 \pm 0.11 \mathrm{ab}$ & $26.96 \pm 0.40^{a}$ & SB \\
\hline & $14.13 \pm 0.13^{b}$ & $0.44 \pm 0.02^{\mathrm{ab}}$ & $0.22 \pm 0.04 a$ & $0.43 \pm 0.01^{c}$ & $3.89 \pm 0.13^{c}$ & $0.35 \pm 0.04 a$ & $0.20 \pm 0.04^{\mathrm{ab}}$ & $5.81 \pm 0.07 c$ & $25.45 \pm 0.21^{c}$ & Corn \\
\hline & $14.10 \pm 0.02^{\mathrm{b}}$ & $0.52 \pm 0.03^{a}$ & $0.19 \pm 0.00^{a}$ & $0.45 \pm 0.01^{\mathrm{c}}$ & $3.93 \pm 0.06^{\mathrm{bc}}$ & $0.34 \pm 0.02^{\mathrm{ab}}$ & $0.19 \pm 0.01^{b}$ & $6.20 \pm 0.08 \mathrm{bc}$ & $25.90 \pm 0.06^{\mathrm{bc}}$ & 0 \\
\hline \multirow{4}{*}{10} & $13.25 \pm 0.21^{b}$ & $0.45 \pm 0.01^{\mathrm{ab}}$ & $0.23 \pm 0.00^{a}$ & $0.53 \pm 0.00^{a}$ & $3.12 \pm 0.02^{c}$ & $0.41 \pm 0.02^{\mathrm{ab}}$ & $0.22 \pm 0.00^{\mathrm{ab}}$ & $4.32 \pm 0.11 \mathrm{e}$ & $22.51 \pm 0.31^{\mathrm{d}}$ & C \\
\hline & $13.75 \pm 0.20^{\mathrm{ab}}$ & $0.46 \pm 0.01^{\mathrm{ab}}$ & $0.18 \pm 0.01^{b}$ & $0.42 \pm 0.01^{b}$ & $3.56 \pm 0.16^{\mathrm{ab}}$ & $0.36 \pm 0.04^{b c}$ & $0.19 \pm 0.01^{b}$ & $5.73 \pm 0.07 \mathrm{bcd}$ & $24.63 \pm 0.23^{c}$ & $S$ \\
\hline & $13.92 \pm 0.04^{\mathrm{ab}}$ & $0.46 \pm 0.01^{\mathrm{ab}}$ & $0.22 \pm 0.02^{\mathrm{ab}}$ & $0.47 \pm 0.06^{\mathrm{ab}}$ & $3.36 \pm 0.21 \mathrm{bc}$ & $0.34 \pm 0.04 \mathrm{bc}$ & $0.20 \pm 0.03^{\mathrm{ab}}$ & $5.95 \pm 0.13 \mathrm{abc}$ & $24.91 \pm 0.14^{b c}$ & H \\
\hline & $14.12 \pm 0.08^{\mathrm{ab}}$ & $0.33 \pm 0.17 \mathrm{~b}$ & $0.21 \pm 0.02^{\mathrm{ab}}$ & $0.47 \pm 0.06^{\mathrm{ab}}$ & $3.71 \pm 0.07^{a}$ & $0.31 \pm 0.01^{c}$ & $0.19 \pm 0.01^{b}$ & $6.01 \pm 0.11 \mathrm{ab}$ & $25.33 \pm 0.13^{b}$ & Can \\
\hline
\end{tabular}




\begin{tabular}{|c|c|c|c|c|c|c|c|c|c|c|}
\hline & $15.20 \pm 0.14^{a}$ & $0.50 \pm 0.02^{\mathrm{ab}}$ & $0.23 \pm 0.01^{a}$ & $0.50 \pm 0.01^{\mathrm{ab}}$ & $3.43 \pm 0.16^{a b}$ & $0.35 \pm 0.03^{b c}$ & $0.22 \pm 0.01^{\mathrm{ab}}$ & $6.17 \pm 0.04^{a}$ & $26.59 \pm 0.03^{\mathrm{a}}$ & SB \\
\hline & $14.07 \pm 0.29 \mathrm{ab}$ & $0.45 \pm 0.02^{\mathrm{ab}}$ & $0.23 \pm 0.00^{\mathrm{a}}$ & $0.50 \pm 0.03^{\mathrm{ab}}$ & $3.68 \pm 0.04^{a}$ & $0.32 \pm 0.06^{b c}$ & $0.23 \pm 0.00^{a}$ & $.06^{\mathrm{cd}}$ & $25.06 \pm 0.31 \mathrm{bc}$ & Corn \\
\hline & $14.08 \pm 0.06^{a b}$ & $0.54 \pm 0.02^{a}$ & $0.22 \pm 0.03 \mathrm{ab}$ & $0.49 \pm 0.06^{a b}$ & $3.69 \pm 0.09 \mathrm{a}$ & $0.48 \pm 0.04 a$ & $0.20 \pm 0.02^{\mathrm{ab}}$ & $5.51 \pm 0.35^{d}$ & $25.18 \pm 0.06^{b}$ & 0 \\
\hline & $12.34 \pm 0.37 c$ & $0.41 \pm 0.01^{b}$ & $0.21 \pm 0.03^{a}$ & $0.55 \pm 0.03^{a}$ & $2.81 \pm 0.12^{c}$ & $0.38 \pm 0.04^{a}$ & $0.18 \pm 0.01^{a}$ & $4.06 \pm 0.08 \mathrm{e}$ & $20.94 \pm 0.47 c$ & C \\
\hline & $13.48 \pm 0.10^{b}$ & $0.44 \pm 0.01^{\mathrm{ab}}$ & $0.21 \pm 0.03^{a}$ & $0.47 \pm 0.06^{\mathrm{ab}}$ & $3.48 \pm 0.05^{\mathrm{ab}}$ & $0.34 \pm 0.02^{a}$ & $0.21 \pm 0.04^{a}$ & $5.69 \pm 0.06^{b}$ & $0.27^{b}$ & S \\
\hline & $13.36 \pm 0.16^{b}$ & $0.44 \pm 0.02^{\mathrm{ab}}$ & $0.20 \pm 0.01 \mathrm{a}$ & $0.42 \pm 0.00^{b}$ & $3.23 \pm 0.12^{b}$ & $0.27 \pm 0.01 \mathrm{a}$ & $0.17 \pm 0.01 \mathrm{a}$ & $5.63 \pm 0.07 \mathrm{bc}$ & $23.70 \pm 0.01 \mathrm{~b}$ & H \\
\hline & $13.49 \pm 0.28^{b}$ & $0.43 \pm 0.03 \mathrm{ab}$ & $0.21 \pm 0.02^{\mathrm{a}}$ & $0.47 \pm 0.04^{\mathrm{ab}}$ & $3.62 \pm 0.16^{\mathrm{a}}$ & $0.27 \pm 0.04^{a}$ & $0.20 \pm 0.02^{\mathrm{a}}$ & $5.79 \pm 0.20^{\mathrm{ab}}$ & $24.46 \pm 0.01^{b}$ & Can \\
\hline & $14.87 \pm 0.04^{a}$ & $0.47 \pm 0.03^{a}$ & $0.20 \pm 0.03^{a}$ & $0.49 \pm 0.06^{\mathrm{ab}}$ & $3.28 \pm 0.08 \mathrm{ab}$ & $0.97 \pm 0.35^{a}$ & $0.18 \pm 0.03^{a}$ & $5.96 \pm 0.12^{\mathrm{a}}$ & $26.41 \pm 1.08^{a}$ & SB \\
\hline & $13.45 \pm 0.28^{b}$ & $0.44 \pm 0.01 \mathrm{ab}$ & $0.20 \pm 0.01^{\mathrm{a}}$ & $0.46 \pm 0.02^{\mathrm{ab}}$ & $3.45 \pm 0.16 \mathrm{ab}$ & $0.30 \pm 0.01^{\mathrm{a}}$ & $0.19 \pm 0.02^{\mathrm{a}}$ & $5.31 \pm 0.08^{d}$ & $23.78 \pm 0.12^{b}$ & Corn \\
\hline & $13.81 \pm 0.08^{b}$ & $0.48 \pm 0.02^{a}$ & $0.25 \pm 0.04 a$ & $0.55 \pm 0.08^{a b}$ & $3.35 \pm 0.21 \mathrm{ab}$ & $0.39 \pm 0.02^{\mathrm{a}}$ & $0.20 \pm 0.02^{\mathrm{a}}$ & $5.45 \pm 0.05^{c d}$ & $24.48 \pm 0.06^{b}$ & 0 \\
\hline
\end{tabular}

Means sharing the same letter in the same row $(a-e)$ are not significantly different $(p>0.05)$ using Duncan's multiple range test. C: Control, S: sunflower, $\mathrm{H}$ : hazelnut, Can: Canola, SB: Soybean, Corn: Corn, O: Olive.

Eicosapentaenoic acid (EPA) is one of the most important polyunsaturated fatty acids for human health. EPA level was found as $4.21 \%$ at the beginning of the storage period, while it was measured as $2.81 \%, 3.48 \%, 3.23 \%, 3.62 \%, 3.28 \%, 3.45 \%$, $3.35 \%$ at the end of the storage period for the control, sunflower, hazelnut, canola, soybean, corn and olive oil groups respectively. Statistically significant differences were observed between the control group and treatment groups during the storage period $(p<0.05)$. The highest EPA level was found in the corn oil group on day 2 of storage as $4.73 \%$. The lowest EPA level was found in the control group at the end of the storage period as $2.81 \%$. While there were no statistically significant differences between treatment groups on the second day of storage, a statistically significant difference was found with the control group $(p<0.05)$. While no statistically significant differences were observed between soybean and corn oil groups on storage day 8 , statistically significant differences were observed between all groups and the control group $(p<0.05)$. Ozogul at al. $(2017)$ reported that the EPA values of sea bass (Dicentrarchus labrax) fillets treated with commercial oil nanoemulsions decreased during the storage period. On the other hand, the EPA values of the groups treated with nanoemulsions were higher than the control group throughout the storage period. A similar decrease was observed in present study. Özoğul et al. (2007) reported that the EPA values of commercially valuable species present in Turkish seas were between $4.74 \%$ and $11.7 \%$.

DHA is another very important polyunsaturated fatty acid. At the beginning of the storage period, DHA value was found as $8.09 \%$ and this is the highest DHA value recorded during the storage period. This value decreased during storage period and statistically significant differences were observed between groups. At the end of the storage period, DHA value was found as $4.06 \%, 5.69 \%, 5.63 \%, 5.79 \%, 5.96 \%, 5.31 \%, 5.45 \%$ for the control, sunflower, hazelnut, canola, soybean, corn and olive oil nanoemulsion groups respectively. At the end of the storage period, the highest DHA level was found in the soybean oil group with $5.96 \%$, while the lowest DHA level was found in the control group with $4.06 \%$. During the storage period, it was determined that the DHA levels were higher in the treatment groups compared to the control group. Alasalvar et al. (2002) reported that the DHA values for wild caught and cultured bass were $19.5 \%$ and $18.1 \%$ respectively. Özoğul et al. (2007) reported that the DHA value in sea bass fillets was $14.7 \%$. Lenas et al. (2011) reported the DHA level of wild caught bass as $17.17 \%$ and cultured bass as $8.62 \%$. The DHA level found in present study was similar to the value of sea bass found by Lenas et al. (2011).

Fatty acid composition of sea bass was significantly influenced by nanoemulsion application $(p<0.05)$. In general, the nanoemulsion treatment groups were found to have higher MUFA and PUFA contents than those of the control group. Among the nanoemulsion treatment groups, the highest PUFA value in the soybean group was observed and the lowest value in the hazelnut group was observed at the end of storage.

\section{CONCLUSIONS}

At the end of the storage period, saturated fatty acid levels in the control and nanoemulsion treatment groups increased and monounsaturated and polyunsaturated fatty acid levels decreased. Most researchers associate this decrease with the transformation of especially unsaturated fatty acids among fatty acids becoming free as a result of lipolysis reactions to first peroxides and secondarily to aldehydes, ketones, alcohols and esthers as a result of auto-oxidation reactions. In the present study, lowest polyunsaturated fatty acid levels were found in the control group on the last day of the storage period. Among nanoemulsion groups, soybean oil group gave the highest PUFA content. It was thought that the application of nanoemulsions delay the auto-oxidation reactions of unsaturated fatty acids. As a result, it was thought that the type of oil used to prepare nanoemulsions can have a positive effect on fatty acid compositions.

\section{ACKNOWLEDGMENTS}

The authors wish to thank the Scientific and Technological Research Council of Turkey (TÜBITAK) for the financial support (TOVAG-1130379) and the authors also gratefully acknowledge infrastructure support provided by Cukurova University. 


\section{REFERENCES}

Al-Adham, I.S.I., Khalil, E., Al-Hmoud, N.D., Kierans, M. \& Collier, P.J. (2000) Microemulsions are membrane-active, antimicrobial, self-preserving systems. Journal of Applied Microbiology, 89(1): 32-39. DOI: 10.1046/j.1365-2672.2000.01078.x

Alasalvar, C., Taylor, K.D.A., Öksüz, A., Garthwaite, T., Alexis, M.N. \& Grigorakis, K. (2001). Freshness assessment of cultured sea bream (Sparus aurata) by chemical, physical and sensory methods. Food Chemistry, 72(1):33-40. DOI: 10.1016/S0308-8146(00)00196-5

Anton, N., Benoit, J.P. \& Saulnier, P. (2008). Design and production of nanoparticles formulated from nano-emulsion templates-a review. Journal of Controlled Release, 128(3):185-199. DOI: 10.1016/j.jconrel.2008.02.007

Ba, H.V., Touseef, A. \& Hwang, I. (2013). Significant influence of particular unsaturated fatty acids and $\mathrm{pH}$ on the volatile compounds in meat-like model system. Meat Science, 94: 480-488. DOI: 10.1016/j.meatsci.2013.04.029

Baki, B., Gönener, S. \& Kaya, D. (2015). Comparison of food, amino acid and fatty acid compositions of wild and cultivated sea bass (Dicentrarchus labrax L., 1758). Turkish Journal of Fisheries and Aquatic Sciences, 15(1):175-179. DOI: 10.4194/1303-2712-v15_1_19

Bligh, E.G. \& Dyer, W.J. (1959). A rapid method of total lipid extraction and purification. Canadian journal of biochemistry and physiology, 37(8): 911 917.

Bortoleto, R.K., De Oliveira, A.H.C., Ruller, R., Arni, R.K. \& Ward, R.J. (1998) Tertiary Structural Changes of the a-Hemolysin from Staphylococcus aureuson Association with Liposome Membranes. Archives of biochemistry and biophysics, 351(1):47-52. DOI: 10.1006/abbi.1997.0550

Bouchemal, K., Briançon, S., Perrier, E. \& Fessi, H. (2004). Nano-emulsion formulation using spontaneous emulsification: solvent, oil and surfactan optimisation. International journal of pharmaceutics, 280(1-2):241-251. DOI: 10.1016/j.jpharm.2004.05.016

Chepurnov, A.A., Bakulina, L.F., Dadaeva, A.A., Ustinova, E.N., Chepurnova T. S. \& Baker Jr, J.R. (2003). Inactivation of Ebola virus with a surfactant nanoemulsion. Acta tropica, 87(3): 315-320. DOI: 10.1016/S0001-706X(03)00120-7

Constantinides, P.P., Chaubal, M.V. \& Shorr, R. (2008). Advances in lipid nanodispersions for parenteral drug delivery and targeting. Advanced Drug Delivery Reviews, 60(6):757-767. DOI: 10.1016/j.addr.2007.10.013

Donovan, B.W., Reuter, J.D., Cao, Z., Myc, A., Johnson, K.J. \& Baker Jr, J.R (2000). Prevention of murine influenza A virus pneumonitis by surfactant nano-emulsions. Antiviral Chemistry and Chemotherapy, 11(1), 41-49.

Durmus, M. (2016). Effects of Nanoemulsion Based on Plant Oils on Sensory, Chemical and Microbiological Quality of Sea Bass Fillets (Dicentrarchus Labrax) under Chilled $\left(2 \pm 2^{\circ} \mathrm{C}\right)$ and Vacuum Packed Conditions. PhD Thesis, Department of Fisheries and Processing Technology, Institute of Natural and Applied Science, Cukurova University, 203.

Fathi, M., Mozafari, M.R. \& Mohebbi, M. (2012). Nanoencapsulation of food ingredients using lipid based delivery systems. Trends in Food Science \& Technology, 23(1):13-27. DOl:10.1016/j.tifs.2011.08.003

Friberg, S.E. (1984). Microemulsions in relation to cosmetics and their preservation. Cosmetic and drug preservation, principles and practice, cosmetic science and technology series, 1.

Guo, H., Wilking, J.N., Liang, D., Mason, T.G., Harden, J.L. \& Leheny, R.L. (2007). Slow, nondiffusive dynamics in concentrated nanoemulsions. Physical Review E, 75(4): 041401.

Halliwell, B., Murcia, M.A., Chirico, S. \& Aruoma, O.I. (1995). Free radicals and antioxidants in food and in vivo: What they do and how they work. Critical Reviews in Food Science and Nutrition, 35:7-20. DOI: $10.1080 / 10408399509527682$
Hamouda, T., Hayes, M.M., Cao, Z., Tonda, R., Johnson, K., Wright, D.C. \& Baker Jr, J.R. (1999). A novel surfactant nanoemulsion with broadspectrum sporicidal activity against Bacillus species. Journal of Infectious Diseases, 180(6):1939-1949. DOI: 10.1086/315124

Ichihara, K.I., Shibahara, A., Yamamoto, K. \& Nakayama, T. (1996). An improved method for rapid analysis of the fatty acids of glycerolipids. Lipids, 31(5):535-539. DOI: 10.1007/BF02522986

Itsiopoulos, C., Hodge, A. \& Kaimakamis, M. (2009). Can the Mediterranean diet prevent prostate cancer? Molecular Nutrition \& Food Research, 53(2):227-239. DOI: 10.1002/mnfr.200800207

Joe, M.M., Chauhan, P.S., Bradeeba, K., Shagol, C., Sivakumaar, P.K. \& Sa, T. (2012). Influence of sunflower oil based nanoemulsion (AUSN-4) on the shelf life and quality of Indo-Pacific king mackerel (Scomberomorus guttatus) steaks stored at $20 \mathrm{C}$. Food Control, 23(2): 564-570. DOI: 10.1016/j.foodcont.2011.08.032

Jones, M.N., Song, Y.H., Kaszuba, M. \& Reboiras, M.D. (1997). The interaction of phospholipid liposomes with bacteria and their use in the delivery of bactericides. Journal of drug targeting, 5(1): 25-34. DOI: 10.3109/10611869708995855

Kaya, Y., Duyar, H.A. \& Erdem, M.E. (2004). The importance of fish fatty acids on human health. Ege Journal of Fisheries and Aquatic Sciences, 21(3/4): 365-370.

Ke, P.J. \& Ackman, R. G. (1973). Bunsen coefficient for oxygen in marine oils at various temperatures determined by exponential dilution method with a polarographic oxygenelectrode. Journal of the American Oil Chemists' Society, 50: 429-435

Kinsella, J.E. (1987). Seafoods and fish oils in human health and disease. M. Dekker. Inc. New York, 231-236.

Leaf, A. \& Weber, P.C. (1988). Cardiovascular Effects of n-3 Fatty Acids. New England Journal of Medicine, 318(9):549-557.

Lenas, D., Chatziantoniou, S., Nathanailides, C. \& Triantafillou, D. (2011). Comparison of wild and farmed sea bass (Dicentrarchus labrax $\mathrm{L}$ ) lipid quality. Procedia Food Science, 1: 1139-1145. DOI:10.1016/j.profoo.2011.09.170

Liu, W., Sun, D., Li, C., Liu, Q. \& Xu, J. (2006). Formation and stability of paraffin oil-in-water nano-emulsions prepared by the emulsion inversion point method. Journal of Colloid and Interface Science, 303(2):557-563.

DOI: 10.1016/j.jcis.2006.07.055

Llauradó, E., Albar, S.A., Giralt, M., Solà, R. \& Evans, C.E.L. (2016). The effect of snacking and eating frequency on dietary quality in British adolescents. European journal of nutrition, 55(4): 1789-1797. DOI: $10.1007 / \mathrm{s} 00394-015-0997-8$

Mason, T.G., Wilking, J.N., Meleson, K., Chang, C.B. \& Graves, S.M. (2006). Nanoemulsions: formation, structure, and physical properties. Journal of Physics: Condensed Matter, 18(41): R635

Meleson, K., Graves, S. \& Mason, T.G. (2004). Formation of concentrated nanoemulsions by extreme shear. Soft Materials, 2(2-3):109-123. DOI: $10.1081 /$ SMTS-200056102

Myc, A., Vanhecke, T., Landers, J.J., Hamouda, T. \& Baker, J.R. (2003). The fungicidal activity of novel nanoemulsion (X8W $60 \mathrm{PC}$ ) against clinically important yeast and filamentous fungi. Mycopathologia, 155(4):195-201.

Nuernberg, K., Fischer, K., Nuernberg, G., Kuechenmeister, U., Klosowska, D. \& Eliminowska-Wenda, G. (2005). Effect of dietary olive and linseed oil on lipid composition, meat quality, sensory characteristic and muscle structure in pigs. Meat Science, 70:63-74. DOI: 10.1016/j.meatsci.2004.12.001

Ozden, O. \& Erkan, N. (2010). Impacts of gamma radiation on nutritional components of minimal processed cultured sea bass (Dicentrarchus labrax). Iranian Journal of Fisheries Sciences, 9(2):265-278. 
Ozogul, Y., Durmus, M., Uçar, Y., Köşker, A.R. \& Ozogul, F. (2017). The combined impact of nanoemulsion based on commercial oils and vacuum packing on the fatty acid profiles of sea bass fillets. Journal of Food Processing and Preservation, 41(6). DOI: 10.1111/ffpp.13222

Özogul, Y., Durmus, M., Ucar, Y., Özogul, F. \& Regenstein, J.M. (2016) Comparative study of nanoemulsions based on commercial oils (sunflower, canola, corn, olive, soybean, and hazelnut oils): Effect on microbial, sensory, and chemical qualities of refrigerated farmed sea bass. Innovative Food Science \& Emerging Technologies, 33:422-430. DOI: 10.1016/j.ffset.2015.12.018

Özogul, Y., Özogul, F. \& Alagoz, S. (2007). Fatty acid profiles and fat contents of commercially important seawater and freshwater fish species of Turkey: A comparative study. Food chemistry, 103(1): 217-223. DOI:10.1016/j.foodchem.2006.08.009

Palmquist, D.L. (2009). Omega-3 fatty acids in metabolism, health, and nutrition and for modified animal product foods. The Professional Animal Scientist, 25(3): 207-249. DOI: 10.15232/S1080-7446(15)30713-0

Periago, M.J., Ayala, M.D., López-Albors, O., Abdel, I., Martinez, C., GarcíaAlcázar, A. \& Gil, F. (2005). Muscle cellularity and flesh quality of wild and farmed sea bass, Dicentrarchus labrax L. Aquaculture, 249(1-4): 175-188. DOI:10.1016/i.aquaculture.2005.02.047

Porras, M., Solans, C., Gonzalez, C. \& Gutierrez, J.M. (2008). Properties of water-in-oil (W/O) nano-emulsions prepared by a low-energy emulsification method. Colloids and Surfaces A: Physicochemical and Engineering Aspects, 324(1-3): 181-188. DOI: 10.1016/j.colsurfa.2008.04.012

Rendeiro, C., Sheriff, A., Bhattacharya, T.K., Gogola, J.V., Baxter, J.H., Chen, H. \& Rhodes, J.S. (2016). Long-lasting impairments in adult neurogenesis, spatial learning and memory from a standard chemotherapy regimen used to treat breast cancer. Behavioural Brain Research, 315: 10-22. DOI: 10.1016/j.bbr.2016.07.043

Rossi, J. \& Leroux, J.C. (2007). Principles in the development of intravenous lipid emulsions. Role of Lipid Excipients in Modifying Oral and Parenteral Drug Delivery. Hoboken, New Jersey: Wiley-Interscience, 88-123.

Russel, W.B., Saville, D.A. \& Schowalter, W.R. (1989). Colloidal dispersions. Cambridge university press.

Saglık, S., Alpaslan, M., Gezgin, T., Çetintürkc, K., Tekinay, A. \& Güven, K.C. (2003). Fatty acid composition of wild and cultivated gilthead seabream (Sparus aurata) and sea bass (Dicentrarchus labrax). European Journal of Lipid Science and Technology,105(2): 104-107. DOI: 10.1002/ejlt.200390013

Sakulku, U., Nuchuchua, O., Uawongyart, N., Puttipipatkhachorn, S. Soottitantawat, A. \& Ruktanonchai, U. (2009). Characterization and mosquito repellent activity of citronella oil nanoemulsion. International Journal of Pharmaceutics,372(1-2):105-111. DOI: $10.1016 /$ j.ijpharm.2008.12.029
Shadman, S., Hosseini, S.E., Langroudi, H.E. \& Shabani, S. (in press). Evaluation of the effect of a sunflower oil-based nanoemulsion with Zataria multiflora Boiss. essential oil on the physicochemical properties of rainbow trout (Oncorhynchus mykiss) fillets during cold storage. LWT - Food Science and Technology. DOI: 10.1016/j.Iwt.2016.01.073

Shakeel, F. \& Ramadan, W. (2010). Transdermal delivery of anticancer drug caffeine from water-in-oil nanoemulsions. Colloids and Surfaces $B$ : Biointerfaces, 75(1): 356-362. DOI: 10.1016/j.colsurfb.2009.09.010

Simopoulos, A.P. (1991). Omega-3 fatty acids in health and disease and in growth and development. The American Journal of Clinical Nutrition, 54(3):438-463. DOI: 10.1093/ajcn/54.3.438

Solans, C., Esquena, J., Forgiarini, A.M., Uson, N., Morales, D., Izquierdo, P. \& Garcia-Celma, M.J. (2003). Absorption and aggregation of surfactants in solution. Nano-emulsions: Formation, Properties and applications. Marcel Dekker, New York, 525-554.

Stoll, B.A. (2002). N-3 fatty acids and lipid peroxidation in breast cancer inhibition. British Journal of Nutrition, 87(3):193-198. DOI: 10.1079/BJN2001512

Tadros, T., Izquierdo, P., Esquena, J., \& Solans, C. (2004). Formation and stability of nano-emulsions. Advances in Colloid and Interface Science, 108:303-318

Talegaonkar, S., Mustafa, G., Akhter, S. \& Iqbal, Z.I. (2010). Design and development of oral oil-in-water nanoemulsion formulation bearing atorvastatin: in vitro assessment. Journal of Dispersion Science and Technology, 31(5):690-701. DOI: 10.1080/01932690903120540

Türkkan, A.U., Cakli, S. \& Kilinc, B. (2008). Effects of cooking methods on the proximate composition and fatty acid composition of seabass (Dicentrarchus labrax, Linnaeus, 1758). Food and Bioproducts Processing, 86(3):163-166.

Unger, E.C., Porter, T., Culp, W., Labell, R., Matsunaga, T. \& Zutshi, R. (2004). Therapeutic applications of lipid-coated microbubbles. Advanced Drug Delivery Reviews, 56(9), 1291-1314. DOI: 10.1016/j.addr.2003.12.006

Yazgan, H. (2013). "Effects of Nanoemulsion Based on Sunflower Oil on Sensory, Chemical and Microbiological Quality of Sea Bass (Dicentrarchus Labrax) and Sea Bream (Sparus aurata) Stored at Chilled Temperature $\left(2 \pm 2^{\circ} \mathrm{C}\right)$ ", $\mathrm{PhD}$ Thesis, Department of Fisheries and Processing Technology, Institute of Natural and Applied Science, Cukurova University, 100.

Yildiz, M., Sener, E. \& Timur, M. (2008). Effects of differences in diet and seasonal changes on the fatty acid composition in fillets from farmed and wild sea bream (Sparus aurata L.) and sea bass (Dicentrarchus labrax L.) International Journal of Food Science \& Technology, 43(5):853-858. DOI: 10.1111/j.1365-2621.2007.01526.x

Zhang, H., Lu, Z., Zhang, L., Bao, Y., Zhan, X., Feng, F. \& Zheng, X. (2008). Antifungal activity of a food-grade dilution-stable microemulsion against Aspergillus niger. Letters in Applied Microbiology, 47(5): 445-450. DOI: 10.1111/j.1472-765X.2008.02437.x 